\title{
O.S.P.
}

\section{Le rôle d'un faible contrôle des émotions et de la réussite scolaire dans le chômage de longue durée}

Role of low self-control of emotions and academic achievement in selection into long-term unemployment

\section{Katja Kokko}

Traducteur : Alain Frangi et Pascal Mallet

\section{(2) OpenEdition}

\section{Journals}

Édition électronique

URL : https://journals.openedition.org/osp/645

DOI : $10.4000 /$ osp.645

ISSN : 2104-3795

Éditeur

Institut national d'étude du travail et d'orientation professionnelle (INETOP)

Édition imprimée

Date de publication : 1 septembre 2005

Pagination : 337-352

ISSN : 0249-6739

\section{Référence électronique}

Katja Kokko, « Le rôle d'un faible contrôle des émotions et de la réussite scolaire dans le chômage de longue durée ", L'orientation scolaire et professionnelle [En ligne], 34/3 | 2005, mis en ligne le 28 septembre 2009, consulté le 18 janvier 2022. URL : http://journals.openedition.org/osp/645; DOI : https://doi.org/10.4000/osp.645

Ce document a été généré automatiquement le 18 janvier 2022.

(c) Tous droits réservés 


\section{Le rôle d'un faible contrôle des émotions et de la réussite scolaire dans le chômage de longue durée}

Role of low self-control of emotions and academic achievement in selection into long-term unemployment

Katja Kokko

Traduction : Alain Frangi et Pascal Mallet

Cet article a été financé par l'Académie des sciences de Finlande et préparé en tant que composante du projet (44858) «Le développement humain et ses facteurs de risque » financé par l'Académie des sciences de Finlande (Finnish Centre of Excellence Programme, 2000-2005) et par une subvention personnelle accordée à Katja Kokko (55289). Je remercie le Dr Minna Puustinen pour ses précieux commentaires sur ce manuscrit, dont la version anglaise a été traduite en français par Alain Frangi et Pascal Mallet.

\section{Contexte de la présente recherche}

1 Cet article se fonde sur les résultats et données théoriques présentés dans ma thèse de doctorat intitulée "Antécédents et conséquences du chômage de longue durée " (Kokko, 2001). Il s'intéresse aux antécédents du chômage de longue durée et s'efforce d'apporter une réponse aux points suivants : 1) les caractéristiques individuelles de l'enfant en tant qu'antécédents de problèmes sérieux dans le domaine du travail ;2) les mécanismes et facteurs de protection opérant entre les caractéristiques individuelles à 8 ans et le chômage de longue durée ultérieur ; 3) le rôle de la réussite scolaire par rapport à celui des caractéristiques individuelles dans le chômage à différents âges. La figure 1 montre un schéma des parcours étudiés et des variables utilisées dans le présent article. La grande flèche (allant des caractéristiques individuelles de l'enfant au chômage de longue durée de l'adulte) renvoie au premier point distingué ci-dessus. Les petites flèches (allant des caractéristiques individuelles de l'enfant au chômage de 
longue durée en passant par la réussite scolaire) et la flèche verticale (partant des facteurs de protection) renvoient au second point. On examine enfin le troisième point : dans quelle mesure les facteurs individuels et les facteurs de formation scolaire contribuent au chômage de longue durée à différents âges (27 et 36 ans). Les résultats pour ces trois points se fondent sur l'Étude longitudinale de la personnalité et du développement social de Jyväskylä, dans laquelle les mêmes individus, âgés de huit ans à l'origine, ont été suivis à 14, 27, 36, et 42 ans (ce dernier âge n'étant pas considéré ici).

Figure 1 / Figure 1

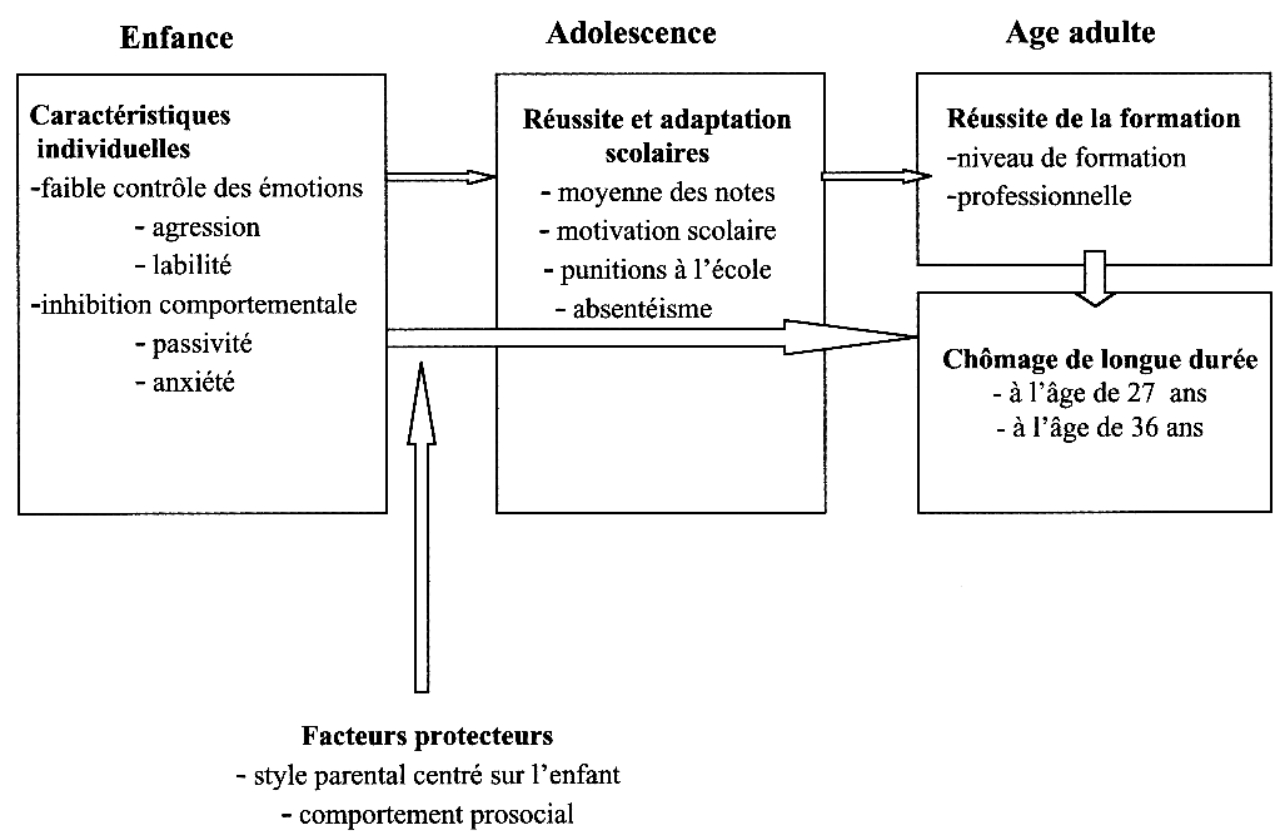

Objectifs de la recherche relative au processus de « sélection » menant au chômage de longue durée / Objectives of the study on the selection process into long-term unemployment.

\section{Introduction}

Outre son coût au niveau sociétal, le chômage est ressenti comme une expérience stressante par les chômeurs et leurs familles (Winefield, 1995). Au niveau individuel, les arguments empiriques abondent qui montrent son association avec la détresse psychologique (par exemple Murphy \& Athanasou, 1999), les problèmes de santé physique (par exemple Wilson \& Walker, 1993), l'abus d'alcool (par exemple Dooley, Catalano \& Hough, 1992) et la délinquance (par exemple Fergusson, Lynskey \& Horwood, 1997). Cet article est centré sur les liens entre l'expérience du chômage et les caractéristiques psychologiques du chômeur.

Traditionnellement, on considère le chômage comme un agent causal de symptômes psychologiques comme la dépression et l'anxiété (Hanisch, 1999 ; Jahoda, 1982 ; Murphy \& Athanasou, 1999 ; Winefield, 1995) bien que cela dépende de la durée du chômage et que les médiateurs en soient, par exemple, les problèmes financiers et une estime de soi diminuée (Kokko \& Pulkkinen, 1998). Une alternative expliquant ce lien entre détresse psychologique et chômage est que la probabilité de se retrouver au chômage est plus forte chez ceux qui ont au préalable des symptômes psychologiques (Rutter \& Rutter, 1993). 
Ces deux hypothèses sur la direction du lien causal entre chômage et détresse psychologique sont dites «hypothèse d'exposition » ou de «causalité sociale " pour la première, et «hypothèse de sélection » ou de « dérive » pour la deuxième (Winefield, 1995, 1997). Il est aussi possible que causalité et sélection opèrent simultanément, c'està-dire que les individus psychologiquement en détresse courent plus de risques de se retrouver au chômage, cette dernière expérience accentuant leur détresse. Un critère minimal pour établir un lien de cause à effet est que les données soient longitudinales (Bergman, Eklund \& Magnusson, 1991). Mortimer (1994) a classifié les études longitudinales consacrées au problème de la «causalité » (au sens défini plus haut) en trois catégories : 1) les études dans lesquelles on prédit le fait d'avoir ou non un emploi ultérieurement à partir de la santé mentale de jeunes terminant leurs études secondaires (par exemple, Hammarström, 1994 ; Tiggemann \& Winefield, 1989) ; 2) les études dans lesquelles, chez des adultes ayant à l'origine un emploi, on s'est efforcé d'identifier des altérations du bien-être à la suite d'une perte d'emploi (par exemple, Dew, Bromet \& Penkower, 1992 ; Hamilton, Hoffman, Broman \& Rauma, 1993) ; et 3) les études qui se sont centrées sur la capacité d'adultes à l'origine sans emploi à retrouver un emploi, ou sur les facteurs expliquant qu'ils restaient sans emploi (par exemple, Warr \& Jackson, 1985).

5 Malgré un nombre accru d'études visant à analyser la validité de ces deux hypothèses, en particulier par rapport au chômage des jeunes (en 1997, le Journal of Adolescence a consacré un numéro entier aux études sur le chômage des jeunes et la détresse psychologique), les conclusions semblent peu claires. Les résultats, qu'ils concernent les jeunes terminant leurs études secondaires ou l'observation de sujets adultes, varient beaucoup dans ce domaine, certains confirmant la «causalité sociale» comme explication - l'expérience sociale du chômage détermine la détresse psychologique (par exemple, Dew et al., 1992 ; Schaufeli, 1997), d'autres étayant à la fois l'hypothèse de la "sélection" (des chômeurs par certaines de leurs caractéristiques individuelles antérieures) et celle du chômage en tant que facteur social de détresse psychologique (par exemple, Hamilton et al., 1993 ; Hammarström \& Janlert, 1997), et d'autres encore soutenant essentiellement l'hypothèse de la sélection (par exemple, Patterson, 1997 ; Schaufeli \& VanYperen, 1992, 1993). Pourtant, Fryer et Winefield (1998) concluent qu'en se fondant sur l'état actuel de la recherche en matière de chômage et de détresse psychologique, c'est la «causalité sociale» plutôt que la sélection par les caractéristiques individuelles qui est le facteur majeur expliquant la relation entre chômage et détresse psychologique. Autrement dit, selon leur revue de question de 1998, il est plus probable que ce soit le chômage qui induise une plus grande détresse, plutôt que ce soit des symptômes psychologiques antérieurs au chômage qui provoquent la perte d'emploi.

6 Les études existantes ont un défaut majeur : elles ne considèrent pas la possibilité que même les jeunes arrêtant les études secondaires puissent avoir eu une expérience du marché du travail, acquise par exemple lors d'un travail comme bénévole au service d'une association ou de petits boulots d'été (Mortimer, 1994). En Finlande, il est possible d'avoir un petit boulot d'été à 15 ans ou même moins. Cependant, il existe des règlements sur le nombre d'heures que peuvent effectuer ces jeunes. Ces expériences de travail sont susceptibles de leur avoir donné une idée de leurs capacités en tant que travailleurs et, en outre, d'avoir affecté leur bien-être psychologique et leur estime de soi. Dans ce cas, il est difficile de tirer des conclusions sur les relations de cause à effet 
entre le chômage et la détresse psychologique. Pour pouvoir tirer des conclusions plus fiables, il faudrait commencer les études de suivi sur les futurs travailleurs suffisamment tôt, avant qu'ils n'aient eu la moindre expérience du marché du travail. Une autre insuffisance de l'utilisation d'échantillons de jeunes quittant l'école au terme de leurs études secondaires est que, du fait qu'une partie des jeunes « décrochent » en cours de scolarité, il se peut qu'ils soient sous-représentés dans ces échantillons alors même qu'ils sont particulièrement exposés au risque du chômage (Caspi, Wright, Moffitt \& Silva, 1998). Le but de cette étude était donc d'examiner le processus de sélection conduisant au chômage en se fondant sur les caractéristiques des individus vers le milieu de leur enfance; les effets possibles du chômage (causalité sociale) ne sont décrits ici que brièvement.

7 L'étude de ces questions a été rendue possible par l'étude finlandaise J.Y.L.S. (Jyväskylä Longitudinal Study of Personality and Social Development) dans laquelle on a suivi les mêmes participants ( $N=369 ; 173$ filles et 196 garçons), âgés à l'origine de 8 ans, aux âges de 14, 27, 36, et 42 ans (Pulkkinen, 2004). On a tiré cet échantillon (né en 1959 et étudié une première fois en 1968) d'une sélection aléatoire de 12 classes complètes de cours élémentaire dans la ville de Jyväskylä, située dans le centre de la Finlande. L'attrition (déperdition des sujets) initiale était de $0 \%$, et le taux de participation est resté élevé pendant toutes les années qu'a duré l'étude. Au moment de la dernière collecte de données en 2001 (42 ans), on a obtenu les informations d'environ $85 \%$ de l'échantillon original. Les résultats présentés ici proviennent des données recueillies en employant des questionnaires relatifs aux expériences de chômage à 27 et à 36 ans. On a décidé de se centrer principalement sur le chômage de longue durée, en considérant soit, à 27 ans, la durée totale de chômage qu'avait connu alors l'individu, soit, à 36 ans, la durée de chômage entre 27 et 36 ans. Les questionnaires d'auto-évaluation utilisés concernaient : l'environnement familial (par exemple, le statut socio-économique et l'atmosphère familiale), la réussite en matière de formation, et d'autres domaines du fonctionnement adulte (par exemple, la consommation d'alcool) à l'âge de 27 ans ; et la détresse psychologique comme une faible estime de soi (mesurée avec l'échelle de Rosenberg, 1965), les symptômes dépressifs (General Behavior Inventory de Depue, 1987), et l'anxiété (Karolinska Scales of Personality de Af Klinteberg, Schalling \& Magnusson, 1986) à l'âge de 36 ans. À l'âge de huit ans, on a utilisé les évaluations faites par les enseignants des conduites sociales des enfants, telles que l'agression physique ou les conduites pro-sociales. Toujours à huit ans, ces mêmes conduites étaient également évaluées par les condisciples, à l'aide de questionnaires sociométriques. Lorsque les participants étaient âgés de 14 ans, on relevait les rapports que les professeurs avaient pu faire concernant des problèmes d'ajustement scolaire, comme l'absentéisme, ainsi que les punitions. On a aussi réuni des informations sur la réussite scolaire (moyenne des notes) à partir des archives des écoles. Il a été démontré que ces méthodes ont de bonnes qualités psychométriques. Pour plus de détails, voir Kokko (2001).

Le chômage est lié à la situation sociétale du moment. Ainsi, il pourrait être important pour les lecteurs de savoir que le chômage était un problème individuel et sociétal sérieux en Finlande pendant les années 1990 à cause d'une récession économique soudaine dans ce pays. Au début des années 1990, le taux de chômage était d'environ $3 \%$ en Finlande; mais il se mit alors à monter soudainement pendant la première moitié des années 1990, atteignant un niveau d'environ 18 \% en 1995 lorsqu'on étudiait les sujets du J.Y.L.S. à 36 ans. Cependant, il semble que le chômage de longue durée ne s'expliquait pas seulement par le taux de chômage sociétal élevé : ceux qui subissaient 
le chômage de longue durée à 36 ans avaient plus de risques aussi d'avoir eu des problèmes d'emploi à 27 ans (en 1986) et d'en avoir à 42 ans (en 2001) (Kokko, 2005), les taux étant d'environ 5 et $10 \%$ pour ces deux dernières années respectivement.

\section{Les caractéristiques individuelles initiales en tant qu'antécédents du chômage de longue durée}

Il n'existe que quelques études longitudinales prospectives dans lesquelles la sélection aboutissant au chômage a été étudiée sur la base des caractéristiques initiales des individus. Dans deux de ces investigations, des études longitudinales prospectives néozélandaises, le Christchurch Health and Development Study (Fergusson, Horwood \& Lynskey, 1997) et le Dunedin Multidisciplinary Health and Development Study (Caspi et al., 1998), on a identifié des marqueurs du chômage ultérieur dans la petite enfance, à huit ans et même à trois ans, respectivement. Fergusson, Horwood et Lyndskey (1997) ont trouvé que les facteurs individuels et familiaux de l'enfance étaient liés au processus de sélection aboutissant au chômage. En particulier, un faible score de Q.I., et des problèmes de comportement à l'âge de 8 ans ; une faible estime de soi et des troubles psychiatriques (par exemple, troubles de l'humeur, du comportement, ou abus d'alcool) ainsi que l'affiliation à des pairs antisociaux, à 15 ans; et une famille au statut socioéconomique peu élevé, un faible niveau d'éducation de la mère et de fréquents conflits entre les parents augmentaient significativement le risque d'une durée prolongée du chômage de 16 à 18 ans. Une fois pris en compte ces effets de sélection, le chômage restait lié à un niveau élevé de troubles psychiatriques, tels que dépression grave, trouble anxieux, trouble des conduites et usage de stupéfiants, mesurés pendant la période allant de 16 à 18 ans. Pourtant, Fergusson, Horwood et Lyndskey (1997) concluent que les troubles psychiatriques dépendent plus de vulnérabilités personnelles que d'expériences ultérieures telles que le chômage.

En accord avec les résultats de Fergusson, Horwood et Lyndskey (1997), Caspi et al. (1998) ont découvert que les problèmes d'ajustement précoces prédisaient un chômage ultérieur. Un tempérament difficile et une faible intelligence, ainsi qu'un statut professionnel peu élevé de la famille, mesurés dès 3 et 5 ans, étaient des antécédents significatifs d'une durée prolongée de chômage entre 15 et 21 ans. De plus, les problèmes de comportement évalués à 7 et 9 ans ainsi que la délinquance et les mauvais résultats scolaires constatés à 15 ans permettaient de prédire le chômage ultérieur de manière significative. Les effets de ces facteurs de risque demeuraient significatifs même lorsqu'on contrôlait la durée des études et la réussite scolaire. Ces deux études longitudinales néo-zélandaises, de type prospectif, qui commencèrent bien avant que les sujets n'aient aucune expérience du marché du travail, montrent qu'il peut y avoir, outre les caractéristiques psychologiques, d'autres facteurs expliquant la sélection qui aboutit au chômage de longue durée.

11 Pour la présente étude, on a mené des investigations sur la sélection aboutissant au chômage à partir des caractéristiques de l'enfant tirées du modèle de régulation émotionnelle et comportementale (Pulkkinen, 1995, 1998 ; connu précédemment comme le modèle du contrôle d'impulsion, voir Pulkkinen, 1982). Ce modèle comporte deux dimensions orthogonales qui correspondent au contrôle des émotions (fort vs. faible) et à l'expression versus l'inhibition du comportement. Ces deux dimensions forment en outre quatre types comportementaux, de A à D. Type A et Type D: comportement 
agressif et anxieux, respectivement ; ils ont en commun un faible contrôle des émotions mais diffèrent quant à l'activité comportementale: l'activité est typique du comportement agressif, alors que la passivité est typique du comportement anxieux. Type $B$ et Type $C$ : comportement constructif et docile, respectivement, les deux présentant un grand contrôle des émotions. Cependant, le comportement constructif se caractérise par l'activité comportementale, alors que le comportement docile se caractérise par la passivité comportementale.

On avait déjà démontré qu'un faible contrôle des émotions pendant l'enfance, et en particulier un comportement agressif, précédait les problèmes d'ajustement ultérieurs. Les résultats indiquent qu'un faible contrôle des émotions est lié à une orientation professionnelle indécise (Pulkkinen, Ohranen \& Tolvanen, 1999), des problèmes d'alcool (Pulkkinen \& Pitkänen, 1994), des arrestations (Pulkkinen \& Hämäläinen, 1995), et un profil de carrière instable chez les hommes (Rönkä \& Pulkkinen, 1995). Le comportement agressif dans la J.Y.L.S., les problèmes de comportement dans l'étude de Fergusson (Fergusson, Horwood \& Lyndskey, 1997 ; Fergusson, Lyndskey \& Horwood, 1997) et les problèmes comportementaux dans celle de Caspi et al. (1998) intègrent tous des composantes de comportements d'agression physique, qui témoignent de l'intention de faire mal à autrui et reflètent une indifférence aux sentiments d'autrui (Loeber \& Hay, 1997). En conséquence, j'étais particulièrement intéressée par le rôle du Type A, le comportement agressif, dans le processus de sélection aboutissant au chômage.

Dans la présente étude, nous avons utilisé l'analyse factorielle des correspondances et nous avons trouvé, de façon convergente avec les recherches précédentes, que la dimension "faible contrôle des émotions " à 8 ans avait une relation plus étroite que l'inhibition comportementale avec le chômage de longue durée à 36 ans (cette dernière variable correspondant à la durée totale au-delà de 2 ans de chômage entre 27 et 36 ans; Kokko, Pulkkinen \& Puustinen, 2000). Le faible contrôle des émotions était caractérisé par le comportement agressif, mais pas par l'anxiété, qui caractérisait la dimension de l'inhibition comportementale. Une modélisation d'équation structurale utilisant un programme LISREL (Jöreskog \& Sörbom, 1996) a indiqué qu'un faible contrôle des émotions à 8 ans avait un lien direct avec le chômage, alors que l'inhibition comportementale était indirectement associée au chômage. "Indirectement associée » signifie que l'inhibition comportementale pendant l'enfance était liée à une faible réussite scolaire à 14 ans, qui à son tour était liée à un faible niveau de formation à 27 ans. De plus, un faible niveau de formation précédait le chômage de longue durée. Ces liens directs et indirects se maintenaient une fois pris en compte les effets du sexe des participants et du statut socio-économique de leurs parents. La part de la variance du chômage de longue durée expliquée était de $7 \%$. Une fois contrôlés le niveau de formation de l'individu et ses autres caractéristiques individuelles qui rendaient compte du chômage de longue durée, on s'est aperçu que ce dernier en lui-même était en relation avec un niveau accru de détresse psychologique, attesté par une faible estime de soi, des symptômes dépressifs et de l'anxiété.

En conséquence, ces résultats ont confirmé les deux hypothèses (causalité et sélection) de la relation entre symptômes psychologiques et chômage. En d'autres termes, il y avait plus de risques que les enfants ayant un faible contrôle de leurs émotions deviennent une fois adultes des chômeurs de longue durée que les enfants n'ayant aucun problème de contrôle de leurs émotions, mais le chômage de longue durée était 
de plus lié à la détresse psychologique. Si l'on a pu trouver des effets de sélection, c'est parce que les participants au J.Y.L.S. avaient été contactés avant qu'ils aient vécu la moindre expérience du marché du travail.

\section{Les mécanismes opérant entre risques et chômage de longue durée}

15 Comme on l'a montré précédemment, la J.Y.L.S. de même que les deux études néozélandaises soutiennent l'idée selon laquelle les problèmes de comportement de l'enfant, tels que les troubles des conduites et les comportements agressif, sont des facteurs de risque pour le chômage de longue durée ultérieur. Il est important de comprendre pourquoi et par quels mécanismes l'agression est un facteur de risque pour un chômage de longue durée ultérieur. On a supposé qu'il existait au moins deux mécanismes pouvant expliquer le risque accru qu'ont les enfants agressifs de se retrouver au chômage: la continuité cumulative et la continuité interactionnelle (Caspi, 1998; Caspi, Bem \& Elder, 1989 ; Caspi, Elder \& Bem, 1987). Par continuité cumulative, Caspi et ses collègues entendent des comportements à partir desquels les individus sélectionnent des environnements qui renforcent encore leurs comportements: par exemple, les comportements agressifs «sont maintenus par l'accumulation progressive de leurs propres conséquences" (Caspi et al., 1987, p. 308). On a montré qu'un comportement agressif durant l'enfance était lié à de mauvaises performances scolaires par la suite (Brook \& Newcomb, 1995 ; Caspi et al., 1987, 1989 ; Rönkä \& Pulkkinen, 1995), lesquelles sont aussi associées au chômage (Caspi et al., 1998 ; Sanford et al., 1994) et à une orientation professionnelle hésitante (Pulkkinen et al., 1999). Des résultats antérieurs ont aussi montré que l'agression chez l'enfant précède des problèmes d'alcool chez l'adulte (Bardone, Moffitt, Caspi, Dickson \& Silva, 1996; Pulkkinen \& Pitkänen, 1994), qui sont à leur tour liés à une faible implication dans le travail (Brook \& Newcomb, 1995), à un profil de carrière instable (Rönkä \& Pulkkinen, 1995) et au chômage (Sanford et al., 1994).

Selon Caspi et al. (1987, 1989), la continuité interactionnelle décrit des comportements maintenus par les réponses réciproques qu'ils provoquent chez autrui. Caspi (1998) a par la suite utilisé l'expression "conséquences contemporaines" pour ce genre de continuité. Il est possible, par exemple, que les individus aux tendances agressives n'aient pas les compétences essentielles pour interagir avec les collègues de travail. De plus, il se peut que des tendances agressives rendent les individus vulnérables au licenciement. Layton et Eysenck (1985) ont suggéré que les individus ayant des tendances hostiles pourraient avoir du mal à se présenter sous un jour favorable à leurs employeurs potentiels, et donc pourraient avoir des problèmes pour retrouver un emploi. À ce jour, la recherche n'a pas réussi à fournir des modèles conceptuels et empiriques adéquats des cheminements allant de l'agression chez l'enfant au chômage de l'adulte. Cependant, certaines études ont fait la lumière sur différentes parties de ces cheminements.

Le but de la présente étude était de combiner ces différentes parties en un seul modèle et, grâce à ce dernier, de rendre compte des mécanismes opérant entre les caractéristiques d'un enfant et un chômage ultérieur. En employant l'échantillon J.Y.L.S. et la modélisation LISREL (qui comporte à la fois une modélisation d'équation structurale et un modèle de mesure), nous avons constaté que le comportement 
agressif durant l'enfance était à l'origine d'un cycle d'inadaptation avec continuité cumulative (Caspi et al., 1987, 1989). Le comportement agressif à 8 ans était lié à l'inadaptation scolaire à 14 ans (attestée par de faibles résultats scolaires, une faible motivation, des punitions et l'absentéisme). Celle-ci était ensuite liée au chômage de longue durée (évalué par la durée totale du chômage au-delà d'une durée de deux ans entre 27 et 36 ans), d'une part directement, et d'autre part indirectement, par l'intermédiaire de problèmes d'alcool et/ou l'absence de possibilités dans le choix d'une profession à l'âge de 27 ans (Kokko \& Pulkkinen, 2000). La part de la variance du chômage de longue durée expliquée était de $25 \%$. Dans cette étude, on n'a pas examiné la continuité interactionnelle, puisqu'elle n'incluait pas les liens synchroniques entre agression et chômage.

\section{Facteurs protecteurs contre le chômage de longue durée chez les enfants agressifs}

Comme dans ce champ de recherche on vient seulement de commencer à identifier les facteurs de risque précoces du chômage et les mécanismes opérant dans cette relation, il n'y avait pas encore d'études des facteurs qui pourraient servir à protéger les personnes ayant un risque élevé de chômage de l'éventualité de se retrouver effectivement sans emploi. Toutefois, dans d'autres domaines que le chômage, on a beaucoup étudié les effets protecteurs, par exemple par rapport aux conséquences de l'agressivité de l'enfant. Les effets protecteurs peuvent être soit personnels soit environnementaux, c'est-à-dire qu'ils viennent de l'individu ou du contexte (Freitas \& Downey, 1998 ; Luthar \& Cicchetti, 2000 ; Masten \& Coatsworth, 1998 ; Rutter, 1985, 1994). On les définit soit comme des processus interactifs ou «tampons » opérant à l'encontre d'un facteur de risque, soit comme des effets principaux (Freitas \& Downey, 1998; Luthar \& Cicchetti, 2000). Si l'on dit d'un certain facteur qu'il a un "effet tampon ", c'est qu'il exerce son influence favorable seulement sur ceux qui présentent le facteur de risque; alors que d'un autre côté, «l'effet principal » est celui qui a des effets positifs aussi bien sur les individus à faible niveau de facteur de risque, que sur ceux ayant un niveau de facteur de risque plus élevé.

Nous nous sommes intéressées dans la présente étude au comportement prosocial en tant que facteur protecteur individuel et au style parental centré sur l'enfant en tant que facteur protecteur environnemental. Il a déjà été démontré que ces deux facteurs exercent aussi bien des effets principaux que des effets tampon sur l'adaptation de l'enfant. En ce qui concerne le comportement prosocial, Hämäläinen and Pulkkinen (1996) ont trouvé qu'en plus de l'agression, le manque de prosocialité dans l'enfance était associé à une délinquance ultérieure. On a montré que les compétences prosociales sont particulièrement utiles à l'enfant agressif : elles le protègent contre l'exclusion par les groupes de pairs (Bierman, Smoot \& Aumiller, 1993 ; Nangle \& Foster, 1992 ; Volling, MacKinnon-Lewis, Rabiner \& Baradaran, 1993). On a aussi trouvé que le rejet par les pairs expliquait l'inadaptation (Cairns \& Cairns, 1994; Magnusson \& Bergman, 1990) et la délinquance (Rutter, Giller \& Hagell, 1998). Dans la présente étude, le comportement prosocial se définit en termes du modèle de régulation émotionnelle et comportementale de Pulkkinen $(1995,1998)$. On a fait l'hypothèse que les individus agressifs mais capables de comportements constructifs s'adaptent mieux aux situations 
critiques de la vie que les individus agressifs n'ayant pas de stratégies constructives pour la résolution des problèmes.

On a déjà montré que le style parental «autoritaire » (Steinberg, Elmen \& Mounts, 1989), ou centré sur l'enfant (Pulkkinen, 1982) - comportant une acceptation parentale, une supervision du comportement et l'octroi d'une autonomie psychologique -, est lié à une bonne réussite scolaire (Steinberg, Lamborn, Dornbusch \& Darling, 1992; Steinberg, Mounts, Lamborn \& Dornbusch, 1991), une confiance en soi élevée, peu de symptômes psychologiques et une absence d'actes de délinquance (Steinberg et al., 1991), ainsi qu'un niveau élevé de contrôle des émotions et une carrière professionnelle stable (Männikkö \& Pulkkinen, 2001). Les résultats suggèrent que le style parental a un effet principal général et positif sur l'adaptation de l'enfant (par exemple, Maccoby, 2000), par exemple en affectant le choix d'un groupe de pairs par l'enfant, son investissement scolaire et les influences des contextes de voisinage sur son développement (par exemple, Collins, Maccoby, Steinberg, Hetherington \& Bornstein, 2000). Toutefois, il se peut que son influence soit particulièrement importante pour les enfants présentant un risque développemental (par exemple, Collins et al., 2000), tel qu'un faible contrôle des émotions. Gottfredson et Hirschi (1990) ont suggéré qu'on peut modifier ce faible contrôle par une éducation efficiente. Dans la présente investigation, je me suis intéressée à l'étude des effets protecteurs possibles du style parental centré sur l'enfant sur la situation ultérieure d'un enfant agressif, en ce qui concerne l'emploi.

21 Nos résultats de la J.Y.L.S., obtenus grâce à une analyse de régression logistique, indiquaient que le comportement prosocial (indiqué par exemple par un comportement constructif et un contrôle des émotions élevé) et le style parental centré sur l'enfant (indiqué par exemple par une bonne relation entre les parents, avec la mère et avec le père) jouaient tous deux un rôle de tampon par rapport à l'éventualité qu'un enfant agressif devienne un adulte chômeur de longue durée à 36 ans (Kokko \& Pulkkinen, 2000). Pour les enfants agressifs, la probabilité de devenir chômeur de longue durée était de $1 \%$ si à la fois la prosocialité et le style parental centré sur l'enfant étaient supérieurs d'un écart-type aux valeurs obtenues pour les enfants d'agressivité moyenne; alors qu'elle était de $45 \%$ s'ils étaient inférieurs d'un écart-type à ces dernières. La prosocialité et le style parental centré sur l'enfant avaient aussi des effets principaux sur le chômage de longue durée, à savoir qu'ils étaient en corrélation négative avec celui-ci pour la totalité de l'échantillon.

\section{Le rôle de la réussite scolaire par rapport aux caractéristiques individuelles dans le chômage à différents âges}

Pour l'instant les résultats de la J.Y.L.S. présentés ici concernent surtout le chômage, en particulier le chômage de longue durée mesuré à 36 ans (et se référant à la période entre 27 et 36 ans). Il serait intéressant de savoir si le chômage de longue durée mesuré plus tôt s'explique aussi par des caractéristiques individuelles précoces et leurs effets cumulés. Les recherches antérieures concernant les effets du chômage ont montré que les chômeurs d'âge mûr, comparés aux jeunes chômeurs, ont une plus grande souffrance psychologique (par exemple, Broomhall \& Winefield, 1990; Rowley \& Feather, 1987). D'après Winefield (1995), il y a au moins deux raisons qui pourraient 
expliquer ces résultats. D'abord, le chômage des jeunes peut différer qualitativement de celui des moins jeunes, car les chômeurs plus âgés ont en général perdu leur travail, alors qu'il est fréquent que les jeunes chômeurs aient des problèmes pour trouver un premier emploi lorsqu'ils ont quitté l'école. Il est possible que la détresse d'être au chômage n'apparaisse pas avant que l'individu n'ait atteint l'âge auquel obtenir du travail est une tâche développementale normative pour sa classe d'âge (voir Baltes, Reese \& Lipsitt, 1980). Cet âge peut cependant varier beaucoup d'un pays ou d'une culture à l'autre, selon leurs systèmes éducatifs respectifs. En second lieu, la détresse psychologique du chômeur adulte peut se confondre avec des problèmes financiers, et il est difficile de séparer ces deux effets.

Nous avons cherché à savoir si, dans la J.Y.L.S. aussi, les antécédents du chômage avaient un poids différent selon l'âge. Dans d'autres études longitudinales prospectives, Fergusson (Fergusson, Horwood \& Lyndskey, 1997 ; Fergusson, Lyndskey \& Horwood, 1997) et Caspi et al. (1998) ont mesuré la durée de chômage chez le jeune adulte à 18 et 21 ans, respectivement. Dans la présente étude, on a évalué cette durée aux âges de 36 et 27 ans et de plus comparé les résultats à 27 ans à ceux de l'étude suédoise I.D.A. (Individual Development and Adaptation; Magnusson \& Bergman, 2000). Dans l'I.D.A., on a évalué le chômage de longue durée à l'âge de 26 ans ; on a aussi mesuré les antécédents au même âge, en utilisant les mêmes types de questions, comme dans la J.Y.L.S.

Selon Rutter (1994), il est essentiel d'essayer de reproduire les résultats des recherches longitudinales avec des échantillons différents. En accord avec cette idée, nous avons fait une recherche sur la sélection aboutissant au chômage en employant un modèle d'équation structurale, pour deux échantillons longitudinaux prospectifs, l'un tiré d'une population finlandaise, l'autre d'une population suédoise. Il n'y avait aucune raison de s'attendre à ce que les résultats concernant la sélection aboutissant au chômage de longue durée à 26 ou 27 ans soient différents pour les sujets finlandais et suédois. Les cultures de ces deux pays, spécialement dans les domaines du système éducatif et de la participation des femmes à l'emploi, sont similaires.

Le but principal était de vérifier si le chômage de longue durée, défini comme ayant duré au moins un an en tout à 27 ans, était aussi lié aux caractéristiques individuelles à 8 ans (Kokko, Bergman \& Pulkkinen, 2003). On a utilisé ces mêmes caractéristiques individuelles, à savoir un faible contrôle des émotions et une inhibition comportementale, et on a testé dans cette étude le même modèle de sélection aboutissant au chômage de longue durée que celui obtenu dans Kokko et al. (2000) pour les chômeurs de 36 ans. Dans l'échantillon I.D.A. suédois, on supposait que les problèmes de comportement et la timidité à 10 ans étaient des indicateurs d'un faible contrôle des émotions et d'une inhibition comportementale, respectivement. Kokko et al. (2003) ont montré que ni le faible contrôle des émotions ni l'inhibition comportementale dans la J.Y.L.S., ni les problèmes de conduite ni la timidité pour l'I.D.A., n'étaient liés directement au chômage de longue durée à l'âge de 26 ou 27 ans. Pourtant ils précédaient une faible réussite scolaire à 14 ans (J.Y.L.S.) ou 13 ans (I.D.A.), qui était ensuite liée à un faible niveau de formation à 27 ans (J.Y.L.S.) ou 26 ans (I.D.A.). Un faible niveau de formation était précédé par un statut socio-économique des parents inférieur. De plus, comme on l'a montré précédemment, ce faible niveau de formation était associé au chômage de longue durée. Les facteurs d'antécédent expliquaient 4 (I.D.A.) à 8 \% (J.Y.L.S.) de la variance pour le chômage de longue durée. En conclusion, à $26 / 27$ ans, on pouvait observer une sélection aboutissant à un parcours 
de formation fondé sur les caractéristiques individuelles de l'enfant, plutôt qu'une sélection aboutissant au chômage de longue durée. Ce résultat a été confirmé par les échantillons de la J.Y.L.S. comme par ceux de l'I.D.A., et à la fois par la modélisation d'équations structurales et par la taille des effets (qui est faible à moyenne pour les caractéristiques individuelles, et importante pour la réussite scolaire).

\section{Conclusions}

Cette revue de question, fondée sur ma thèse de doctorat (Kokko, 2001), vise à montrer que le chômage de longue durée, considéré traditionnellement comme un phénomène purement sociétal, peut s'expliquer aussi par les caractéristiques individuelles précoces. Les facteurs externes tels que les domaines professionnels et les réseaux de liens sociaux des participants n'ont pas été envisagés dans cette étude. Leur inclusion aurait augmenté la proportion de variance expliquée pour le chômage de longue durée. Toutefois, la part de variance expliquée a tout de même une portée significative si l'on considère l'empan d'âge couvert par la présente recherche et le fait qu'elle était centrée sur des variables bien spécifiques. Les caractéristiques individuelles, telles qu'un comportement agressif, constituent peut-être une partie du cycle d'inadaptation dans lequel les problèmes comportementaux de l'enfant sont en relation avec son mauvais ajustement scolaire au cours de l'adolescence, lequel est ensuite - en tant que tel mais aussi indirectement par le biais des problèmes de fonctionnement social chez le jeune adulte - associé au chômage de longue durée chez l'adulte (Kokko \& Pulkkinen, 2000). On a constaté l'existence de facteurs protecteurs, à savoir le style parental centré sur l'enfant et le comportement prosocial, qui atténuaient l'inadaptation ultérieure pour certains enfants agressifs (Kokko \& Pulkkinen, 2000).

Dans cette étude, les caractéristiques de l'enfant avaient une relation plus forte avec le chômage à 36 ans qu'à 27 ans, bien que la durée soit plus longue entre les antécédents et le résultat (Kokko et al., 2003). À 27 ans, il y avait une grande variation du temps que les participants avaient passé sur le marché du travail : ceux qui n'avaient pas continué leurs études après la fin de la scolarité obligatoire à 16 ans pouvaient être sur le marché du travail depuis dix ans, alors que ceux qui avaient continué leurs études en lycée et au-delà étudiaient encore ou venaient de finir leurs études à 27 ans. Seuls les premiers étaient susceptibles de faire l'expérience du chômage. Ainsi, chez les jeunes adultes (moins de 27 ans), ce que la présente recherche explique, c'est le parcours de formation et non le chômage en tant que tel. Une formation de haut niveau semble être un facteur favorisant la stabilité de l'emploi. Une meilleure compréhension de l'individu et des facteurs relatifs à sa formation et expliquant son parcours scolaire et professionnel pourrait aider à identifier les groupes d'enfants qui courent le plus grand risque d'être exclus de la formation et du travail, et pourrait préparer le terrain pour des interventions efficaces destinées à ces individus. Dans la présente recherche, on n'a pas distingué les résultats selon le sexe des individus. À l'avenir, il serait important d'étudier si les facteurs de risque précoces aboutissant à un chômage de longue durée varient selon le sexe. 


\section{BIBLIOGRAPHIE}

Af Klinteberg, B., Scalling, D., \& Magnusson, D. (1986). Self-report assessment of personality traits. Data from the KSP inventory on a representative sample of normal male and female subjects within a developmental project. Report No. 64. Department of Psychology, University of Stockholm.

Baltes, P. B., Reese, R. H., \& Lipsitt, L. P. (1980). Life-span developmental psychology. Annual Review of Psychology,31, 61-110.

Bardone, A. M., Moffitt, T. E., Caspi, A., Dickson, N., \& Silva, P. A. (1996). Adult mental health and social outcomes of adolescent girls with depression and conduct disorder. Development and Psychopathology,8, 811-829.

Bergman, L. R., Eklund, G., \& Magnusson, D. (1991). Studying individual development : Problems and methods. In D. Magnusson, L. R. Bergman, G. Rudinger, \& B. Törestad (Eds.), Problems and methods in longitudinal research: Stability and change (pp. 1-27). Cambridge : Cambridge University Press.

Bierman, K. L., Smoot, D. L., \& Aumiller, K. (1993). Characteristics of aggressive-rejected, aggressive (nonrejected), and rejected (nonaggressive) boys. Child Development,64, 139-151.

Brook, J. S., \& Newcomb, M. D. (1995). Childhood aggression and unconventionality : Impact on later academic achievement, drug use, and workforce involvement. The Journal of Genetic Psychology,156, 393-410.

Broomhall, H. S. \& Winefield, A. H. (1990). A comparison of the affective well-being of young and middle-aged unemployed men matched for length of unemployment. British Journal of Medical Psychology,63, 43-52.

Cairns, R. B. \& Cairns, B. D. (1994). Lifelines and risks : Pathways of youth in our time. Cambridge : Cambridge University Press.

Caspi, A. (1998). Personality development across the life course. In W. Damon (Series Ed.) \& N. Eisenberg (Vol. Ed.), Handbook of child psychology: Vol. 3. Social, emotional, and personality development (pp. 311-388). New York : Wiley.

Caspi, A., Bem, D. J., \& Elder, G. H., Jr. (1989). Continuities and consequences of interactional styles across the life course. Journal of Personality,57, 375-406.

Caspi, A., Elder, G. H., Jr., \& Bem, D. J. (1987). Moving against the world : Life-course patterns of explosive children. Developmental Psychology,23, 308-313.

Caspi, A., Wright, B. R. E., Moffitt, T. E., \& Silva, P. A. (1998). Early failure in the labor market : Childhood and adolescent predictors of unemployment in the transition to adulthood. American Sociological Review,63, 424-451.

Collins, W. A., Maccoby, E. A., Steinberg, L., Hetherington, E. M. \& Bornstein, M. H. (2000). Contemporary research on parenting : The case for nature and nurture. American Psychologist,55, 218-232.

Depue, R. (1987). General Behavior Inventory. Department of Psychology, Cornell University.

Dew, M. A., Bromet, E. J., \& Penkower, L. (1992). Mental health effects of job loss in women. Psychological Medicine,22, 751-764. 
Dooley, D., Catalano, R., \& Hough, R. (1992). Unemployment and alcohol disorder in 1910 and 1990 : Drift versus social causation. Journal of Occupational and Organizational Psychology,65, 277-290.

Fergusson, D. M., Horwood, L. J., \& Lynskey, M. T. (1997). The effects of unemployment on psychiatric illness during young adulthood. Psychological Medicine,27, 371-381.

Fergusson, D. M., Lynskey, M. T., \& Horwood, L. J. (1997). The effects of unemployment on juvenile offending. Criminal Behaviour and Mental Health, 7, 49-68.

Freitas, A. L., \& Downey, G. (1998). Resilience : A dynamic perspective. International Journal of Behavioral Development,22, 263-285.

Fryer, D. \& Winefield, A. H. (1998). Employment stress and unemployment distress as two varieties of labour market induced psychological strain : An explanatory framework. Australian Journal of Social Research,5, 3-18.

Gottfredson, M. R., \& Hirschi, T. (1990). A general theory of crime. Stanford, CA : Stanford University Press.

Hämäläinen, M., \& Pulkkinen, L. (1996). Problem behavior as a precursor of male criminality. Development and Psychopathology, 8, 443-455.

Hamilton, V. L., Hoffman, W. S., Broman, C. L., \& Rauma, D. (1993). Unemployment, distress, and coping : A panel study of autoworkers. Journal of Personality and Social Psychology,65, 234-247.

Hammarström, A. (1994). Health consequences of youth unemployment - review from a gender perspective. Social Science \& Medicine, 38, 699-709.

Hammarström, A. \& Janlert, U. (1997). Nervous and depressive symptoms in a longitudinal study of youth unemployment - selection or exposure ? Journal of Adolescence,20, 293-305.

Hanisch, K. A. (1999). Job loss and unemployment research from 1994 to 1998 : A review and recommendations for research and intervention. Journal of Vocational Behavior,55, 188-220.

Jahoda, M. (1982). Employment and unemployment : A social-psychological analysis. Cambridge : Cambridge University Press.

Jöreskog, K. G. \& Sörbom, D. (1996). LISREL 8 : User's reference guide ( $3^{\text {rd }}$ ed.). Chicago, Il : Scientific Software International, Inc.

Kokko, K. (2001). Antecedents and consequences of long-term unemployment. Jyväskylä Studies in Education, Psychology and Social Research, 183. University of Jyväskylä.

Kokko, K. (2005). Unemployment and psychological distress : Education as a resource factor for employment. Manuscript submitted for publication in L. Pulkkinen, J. Kaprio, \& R. Rose (Eds.), Socioemotional Development and Health from Adolescence to Adulthood. To appear in the Book Series : International Studies on Child and Adolescent Health published by The Cambridge University Press.

Kokko, K., Bergman, L. R., \& Pulkkinen, L. (2003). Child personality characteristics and selection into long-term unemployment in Finnish and Swedish longitudinal samples. International Journal of Behavioral Development,27, 134-144.

Kokko, K. \& Pulkkinen, L. (1998). Unemployment and psychological distress : Mediator effects. Journal of Adult Development, 5, 205-217.

Kokko, K. \& Pulkkinen, L. (2000). Aggression in childhood and long-term unemployment in adulthood : A cycle of maladaptation and some protective factors. Developmental Psychology,36, 463-472. 
Kokko, K., Pulkkinen, L. \& Puustinen, M. (2000). Selection into long-term unemployment and its psychological consequences. International Journal of Behavioral Development,24, 310-320.

Layton, C., \& Eysenck, S. (1985). Psychotism and unemployment. Personality and Individual Differences,6, 387-390.

Loeber, R., \& Hay, D. (1997). Key issues in the development of aggression and violence from childhood to early adulthood. Annual Review of Psychology,48, 371-410.

Luthar, S. S. \& Cicchetti, D. (2000). The construct of resilience : Implications for interventions and social policies. Development and Psychopathology,12, 857-885.

Maccoby, E. E. (2000). Parenting and its effects on children : On reading and misreading behavior genetics. Annual Review of Psychology,51, 1-27.

Magnusson, D., \& Bergman, L. R. (1990). A pattern approach to the study of pathways from childhood to adulthood. In L. Robins \& M. Rutter (Eds.), Straight and devious pathways from childhood to adulthood (pp. 101-115). Cambridge : Cambridge University Press.

Magnusson, D. \& Bergman, L. R. (2000). Individual development and adaptation : The I.D.A. program. In C. G. Janson (Ed.), Seven Swedish longitudinal studies. Stockholm : Swedish Council for Planning and Coordination of Research.

Männikkö, K., \& Pulkkinen, L. (2001). Parenting and personality styles. A long-term longitudinal approach. In J. R. M. Gerris (Ed.), Dynamics of Parenting (pp. 179-195). Leuven, Belgium : GarantUitgevers.

Masten, A. S., \& Coatsworth, J. D. (1998). The development of competence in favorable and unfavorable environments : Lessons from research on successful children. American Psychologist, 53, 205-220.

Mortimer, J. T. (1994). Individual differences as precursors of youth unemployment. In A. C. Petersen \& J. T. Mortimer (Eds.), Youth unemployment and society (pp. 172-198). Cambridge : Cambridge University Press.

Murphy, G. C. \& Athanasou, J. A. (1999). The effect of unemployment on mental health. Journal of Occupational and Organizational Psychology, 72, 83-99.

Nangle, D. W., \& Foster, S. L. (1992). The effects of a positive behavioral context on the social impact of aggressive behavior. Journal of Abnormal Child Psychology,20, 543-553.

Patterson, L. J. M. (1997). Long-term unemployment amongst adolescents : A longitudinal study. Journal of Adolescence,20, 261-280.

Pulkkinen, L. (1982). Self-control and continuity from childhood to adolescence. In B. P. Baltes \& O. G. Brim, Jr. (Eds.), Life-span development \& behavior : Vol. 4 (pp. 63-105). Orlando, FL : Academic Press.

Pulkkinen, L. (1995). Behavioral precursors to accidents and resulting physical impairment. Child Development,66, 1660-1679.

Pulkkinen, L. (1998). Levels of longitudinal data differing in complexity and the study of continuity in personality characteristics. In R. B. Cairns, L. R. Bergman, \& J. Kagan (Eds.), Methods and models for studying the individual (pp. 161-184). Thousand Oaks : Sage Publications.

Pulkkinen, L. (2004). A longitudinal study on social development as an impetus for school reform toward an integrated school day. European Psychologist,9, 125-141. 
Pulkkinen, L. \& Hämäläinen, M. (1995). Low self-control as a precursor to crime and accidents in a Finnish longitudinal study. Criminal Behaviour and Mental Health,5, 424-438.

Pulkkinen, L., Ohranen, M., \& Tolvanen, A. (1999). Personality antecedents of career orientation and stability among women compared to men. Journal of Vocational Behavior,54, 37-58.

Pulkkinen, L., \& Pitkänen, T. (1994). A prospective study of the precursors to problem drinking in young adulthood. Journal of Studies on Alcohol,55, 578-587.

Rönkä, A., \& Pulkkinen, L. (1995). Accumulation of problems in social functioning in young adulthood : A developmental approach. Journal of Personality and Social Psychology,69, 381-391.

Rosenberg, M. (1965). Society and the adolescent self-image. Princeton, NJ : Princeton University Press.

Rowley, K. M. \& Feather, N. T. (1987). The impact of unemployment in relation to age and length of unemployment. Journal of Occupational Psychology, 60, 323-332.

Rutter, M. (1985). Resilience in the face of adversity. Protective factors and resistance to psychiatric disorder. British Journal of Psychiatry, 147, 598-611.

Rutter, M. (1994). Concepts of causation, tests of causal mechanisms, and implications for intervention. In A. C. Petersen \& J. T. Mortimer (Eds.), Youth unemployment and society (pp. 147-171). Cambridge : Cambridge University Press.

Rutter, M., Giller, H., \& Hagell, A. (1998). Antisocial behavior by young people. Cambridge : Cambridge University Press.

Rutter, M. \& Rutter, M. (1993). Developing minds : Challenge and continuity across the life-span. New York : Basic Books.

Rönkä, A., \& Pulkkinen, L. (1995). Accumulation of problems in social functioning in young adulthood : A developmental approach. Journal of Personality and Social Psychology,69, 381-391.

Sanford, M., Offord, D., McLeod, K., Boyle, M., Byrne, C., \& Hall, B. (1994). Pathways into the work force : Antecedents of school and work force status. Journal of the American Academy of Child and Adolescent Psychiatry, 33, 1036-1046.

Schaufeli, W. B. (1997). Youth unemployment and mental health : Some Dutch findings. Journal of Adolescence,20, 281-292.

Schaufeli, W. B. \& VanYperen, N. W. (1992). Unemployment and psychological distress among graduates : A longitudinal study. Journal of Occupational and Organizational Psychology,65, 291-305.

Schaufeli, W. B. \& VanYperen, N. W. (1993). Success and failure in the labour market. Journal of Organizational Behavior,14, 559-572.

Steinberg, L., Elmen, J. D., \& Mounts, N. S. (1989). Authoritative parenting, psychosocial maturity, and academic success among adolescents. Child Development, 60, 1424-1436.

Steinberg, L., Lamborn, S. D., Dornbusch, S. M., \& Darling, N. (1992). Impact of parenting practices on adolescent achievement : Authoritative parenting, school involvement, and encouragement to succeed. Child Development, 63, 1266-1281.

Steinberg, L., Mounts, N. S., Lamborn, S. D., \& Dornbusch, S. M. (1991). Authoritative parenting and adolescent adjustment across varied ecological niches. Journal of Research on Adolescence, 1 , 19-36.

Tiggemann, M. \& Winefield, A. H. (1989). Predictors of employment, unemployment and further study among school-leavers. Journal of Occupational Psychology, 62, 213-221. 
Volling, B. L., MacKinnon-Lewis, C., Rabiner, D., \& Baradaran, L. P. (1993). Children's social competence and sociometric status : Further exploration of aggression, social withdrawal, and peer rejection. Development and Psychopathology, 5, 459-483.

Warr, P. \& Jackson, P. (1985). Factors influencing the psychological impact of prolonged unemployment and re-employment. Psychological Medicine, 15, 795-807.

Wilson, S. H. \& Walker, G. M. (1993). Unemployment and health : A review. Public Health, 107, 153-162.

Winefield, A. H. (1995). Unemployment : Its psychological costs. In C. L. Cooper \& I. T. Robinson (Eds.), International Review of Industrial and Organizational Psychology : Vol. 10 (pp. 169-212). London : Wiley.

Winefield, A. H. (1997). Editorial : Introduction to « The psychological effects of youth unemployment : International perspectives ». Journal of Adolescence, 20, 237-241.

\section{RÉSUMÉS}

Cette revue de question traite du processus de sélection conduisant au chômage de longue durée, en se fondant sur des caractéristiques de personnalité et sur la réussite scolaire des enfants et adolescents, ainsi que sur les problèmes de fonctionnement social chez le jeune adulte (sujet de la thèse de Kokko, 2001). Les résultats s'appuient sur une étude finlandaise (Étude longitudinale de la personnalité et du développement social de Jyväskylä, J.Y.L.S.) dans laquelle les mêmes participants (échantillon d'origine : $\mathrm{N}=369$ ) ont été suivis aux âges de 8, 14, 27, 36 et 42 ans. On y montre qu'à 27 ans, le chômage de longue durée s'explique par un parcours scolaire limité qui est de plus lié à un comportement agressif et anxieux à 8 ans. À 36 ans, c'est-à-dire bien après la période de formation scolaire et professionnelle initiale, les caractéristiques de personnalité de l'enfance, expliquent le processus de sélection aboutissant à un chômage de longue durée plus fortement qu'à 27 ans. Une de ces caractéristiques de personnalité est l'agression physique à huit ans, qui témoigne d'un faible contrôle des émotions et d'une forte activité comportementale. Il est donc important de considérer l'âge des chômeurs pour tirer des conclusions sur les facteurs affectant leurs difficultés dans le domaine du travail.

This review article is focused on describing selection into long-term unemployment on the basis of child and adolescent personality characteristics and academic achievement, as well as problems in social functioning in early adulthood (presented also in Kokko, 2001). The findings are based on the Finnish Jyväskylä Longitudinal Study of Personality and Social Development, where the same participants (original sample: $N=369$ ) have been followed at ages $8,14,27,36$, and 42. It is shown that long-term unemployment at age 27 is explained by low academic education, which is, furthermore, linked to aggressive and anxious behavior at age 8 . When the period of formal education is passed at age 36 , childhood personality characteristics explain the selection into long-term unemployment more strongly than at age 27 . One of these personality characteristics is physical aggression, which reflects a low control of emotions and an intense behavioral activity. Thus, it is important to consider the age of the unemployed person in order to make any conclusions about the factors affecting his or her difficulties in the domain of work. 
INDEX

Keywords : Aggressive behavior, Child-centered parenting, Long-term unemployment, Longitudinal study, Prosocial behavior, Psychological distress

Mots-clés : Attitude parentale centrée sur l'enfant, Chômage de longue durée, Comportement agressif, Comportement prosocial, Détresse psychologique, Étude longitudinale

\section{AUTEURS}

\section{KATJA KOKKO}

Katja Kokko est Chercheuse post-doctorale dans le département de Psychologie de l'Université de Jyväskylä en Finlande. Elle travaille sur le développement de l'identité au cours de l'âge adulte et les facteurs qui, chez l'enfant contribuent au développement de la carrière scolaire et professionnelle à l'adolescence et à l'âge adulte.

Adresser toute correspondance à Katja Kokko : University of Jyväskylä Department of Psychology - P.O. Box 35 (Agora) 40014 University of Jyväskylä Finlande - E-mail : katja.kokko@psyka.jyu.fi Tél : +358142602856 - Fax : +3581426044 